\title{
ADHD and sleep history taking at a resident staffed, university affiliated, community health clinic
}

\begin{abstract}
Objective: To examine healthcare providers' screening practices for obstructive sleep apnea (OSA) in children when considering a diagnosis of attention deficit hyperactivity disorder (ADHD). We hypothesized that patients were not being routinely screened for OSA risk factors prior to being diagnosed with ADHD.
\end{abstract}

Methods: Retrospective chart review examining 113 patient encounters in which a diagnosis of ADHD was given. Children were aged 4 to 18 years of age and were evaluated in a resident staffed, university affiliated community health clinic. We used chi square tests to examine various health care providers' questioning about snoring based on patient demographics, health characteristics, and provider characteristics.

Results: Providers asked about snoring in 15\% of patients. Factors predicting questioning over snoring included provider level $(\mathrm{p}<.01)$ and number of years in training $(\mathrm{p}<.001)$. No patient health factors or provider specialties were significantly predictive.

Conclusion: Results suggest that many children evaluated for ADHD are not being screened for OSA. Among providers, there is wide variation in clinical practice in screening for snoring in children presenting with symptoms of ADHD. Future directions include developing interventions and evidence based methods to increase awareness and screening for OSA in those children with inattention or hyperactivity.

Keywords: attention deficit hyperactivity disorder, obstructive sleep apnea, snoring, sleep medicine

\section{Introduction}

Attention deficit hyperactivity disorder (ADHD) is a disorder manifesting predominantly in childhood with symptoms of hyperactivity, inattention, and impulsivity which can have a dramatic effect on the child's behavioral, social, and emotional functionality. ${ }^{1}$ As many as 8 to $11 \%$ of school aged children are diagnosed with ADHD, and billions of dollars have been dedicated to their care, both pharmacologic and psychological. ${ }^{2}$ The pathogenesis of ADHD is incompletely understood. A genetically inherited imbalance in catecholamine metabolism is the most commonly accepted hypothesis given the available animal studies, functional brain imaging, and patients' efficacious response to stimulant medications. ${ }^{3}$ However, in recent years, a number of researchers have proposed a link between ADHD symptoms and sleep disorders. ${ }^{4-8}$ In a recent review in Pediatrics, Bonuck et al., ${ }^{6}$ found that those with sleep disordered breathing were significantly more likely to have behaviors resembling ADHD than those without sleep disordered breathing. ${ }^{6}$ Obstructive sleep apnea (OSA) is a sleep disorder commonly affecting children and can have significant neuro developmental implications on the child's health. ${ }^{7,8}$ American Academy of Pediatrics clinical practice guidelines recommend that patients or their caregivers be screened for snoring (as well as a focused evaluation for other symptoms if noted to have habitual snoring) at each routine health examination, and if positive, be referred to a sleep specialist. ${ }^{9}$ Despite these recommendations, screening for snoring is widely variable and many children at risk for OSA are not being screened according to the guidelines. ${ }^{10}$ Previous studies have reported screening proportions ranging from $8 \%$ to $24 \%$; however, few studies have examined practitioners' screening patterns for OSA when considering the diagnosis of ADHD. ${ }^{11,12}$ The aims of this study were to describe the snoring screening practices
Volume 8 Issue 4 - 2018

\author{
Shane C Rainey DO,' Natasha BraunBS, \\ Mohannad Mannaa MD $^{3}$ \\ 'Assistant Professor of Clinical Pediatrics, University of Illinois \\ College of Medicine at Peoria, USA \\ ${ }^{2} \mathrm{MD}$ Candidate, University of Illinois College of Medicine at \\ Peoria, USA \\ ${ }^{3}$ Associate Professor of Clinical Pediatrics, Division Head \\ Pediatric Pulmonology and Sleep Medicine,Vice Chair Children's \\ Hospital of Illinois, University of Illinois College of Medicine at \\ Peoria, USA
}

Correspondence: Shane Rainey, University of Illinois College of Medicine at Peoria, 530 NE Glen Oak Ave, Peoria, IL, 61637, Tel I(309) 624-7575, Email Shane.C.Rainey@osfhealthcare.org

Received: August 23, 2018 | Published: August 31, 2018 of various clinicians when considering the diagnosis of ADHD and to analyze various patient and provider characteristics to determine their predictiveness for screening about snoring. We hypothesized that patients were not being routinely screened for OSA risk factors prior to being diagnosed with ADHD.

\section{Methods}

\section{Setting and participants}

This study was a retrospective chart review conducted between January 1st, 2015 and December 31st, 2017. Children aged 4 to 18 years of age presenting with symptoms suggestive of undiagnosed ADHD at either a well-child or illness office visit were eligible. Exclusion criteria included any child with a previous diagnosis of ADHD, ADHD diagnosed prior to January 1st, 2015, any child with a previous sleep evaluation or polysomnography, and any child with a history of adenotonsillectomy. Two office visits were reviewed for each patient: their first office visit with concern for ADHD symptoms, and their first follow up visit thereafter. Encounters were reviewed from four individual clinics under the same healthcare system. Providers included attending physicians, resident physicians, advanced nurse practitioners (APN), and physician assistants (PA). The institutional review board of the University of Illinois College of Medicine at Peoria approved this study and waived informed consent, as no protected health information was collected or stored during the duration of this review.

\section{Data collection and analysis}

Data was collected via retrospective chart review in two encounters per eligible patient during the study time frame. Data was analyzed in 
association with our university's statistician. The primary outcome was the presence of questioning about snoring. Chi-square tests were utilized to analyze bivariate associations between screening for snoring and multiple patient and provider characteristics. The patient characteristics analyzed included gender and body mass index (BMI). Provider characteristics analyzed included provider level (attending, APN, PA, resident) type of training (pediatrician, medicine/pediatrics, family medicine, physician assistant, and nurse practitioner), and number of years in training.

\section{Results}

\section{Patient flow}

Patient flow is illustrated in (Figure 1). 445 unique patient charts were reviewed. 282 patients were diagnosed with ADHD prior to the study time frame. 37 did not have a record of their diagnosis in the electronic medical record, suggesting their diagnostic encounter was completed on paper charts prior to the clinic transitioning to electronic medical record keeping. 4 patients had a previous diagnosis of OSA. 4 patients were diagnosed at an outside, non-affiliated clinic. 2 patients had previous adenotonsillectomy. There were 3 duplicate charts. After these exclusions, 113 patients qualified for inclusion in the study.

\begin{tabular}{|c|c|}
\hline 445 charts reviewed & \multirow{2}{*}{$\begin{array}{l}282 \text { diagnosed before } \\
2015\end{array}$} \\
\hline 163 patients in study & \\
\hline time frame & \multirow{2}{*}{$\begin{array}{l}37 \text { without diagnosis } \\
\text { date }\end{array}$} \\
\hline 126 patients & \\
\hline $\begin{array}{l}\text { diagnosed in study } \\
\text { time frame }\end{array}$ & \multirow{2}{*}{$\begin{array}{l}6 \text { with previous OSA } \\
\text { or T\&A }\end{array}$} \\
\hline 120 patients & \\
\hline $\begin{array}{l}\text { diagnosed and } \\
\text { without slekp }\end{array}$ & \multirow{2}{*}{$\begin{array}{l}4 \text { diagnosed at } \\
\text { outside clinic, } 3 \\
\text { duplicate charts }\end{array}$} \\
\hline disorder & \\
\hline
\end{tabular}

Figure 1 Study Flow Diagram.

\section{Demographics and primary outcome}

Children included in the study numbered 80 males $(71 \%)$ and 33 females (29\%). They were between the ages of 4 and 16, with a mean age of $7.5(\mathrm{SD}=2.6)$ years and a median age of 7 years. Their BMI percentages ranged from 1st percentile to 99th percentile, with a mean BMI at the 69th percentile $(\mathrm{SD}=27)$ and a median at the 77 th percentile. $61 \%$ were diagnosed with ADHD combined subtype (69 patients), 11\% were diagnosed with the hyperactive subtype (12 patients), 12\% were diagnosed with the inattentive subtype (14 patients), and 16 percent had an unspecified ADHD subtype (18 patients). Attending physicians completed $26 \%$ of encounters, APNs and PAs accounted for $9 \%$ of encounters, and resident physicians completed the remaining $65 \%$ of encounters. Of the 113 patients, 17 were screened for snoring $(15 \%)$, while 96 had no questioning about snoring (85\%).

\section{Screening by patient characteristics}

Bivariate analyses were conducted on two major patient characteristics: gender and BMI percentage of the 80 males, 13
(16\%) were screened and $67(84 \%)$ were not screened for snoring. In contrast, of the 33 females, 4 (12\%) were screened and $29(88 \%)$ were not screened for snoring $(P=0.774)$. A BMI threshold was set at the 90th percentile to divide patients into two groups. We hypothesized that a provider's likelihood to question a child about snoring would be greater with increasing BMI, and, although the Centers for Disease Control define an overweight BMI as greater than the 85th percentile to less than the 95th percentile, many providers easily recognize a BMI at the 90th percentile or greater as abnormal in the pediatric population. Of 75 children with a BMI less than the 90th percentile, 61 $(81 \%)$ were not screened and 14 (19\%) were screened for snoring, as opposed to those with a BMI greater than the 90th percentile where 35 $(92 \%)$ were not screened and only $3(8 \%)$ were screened for snoring $(P=0.130)$. Neither patient characteristic were statistically significant in predicting screening for snoring.

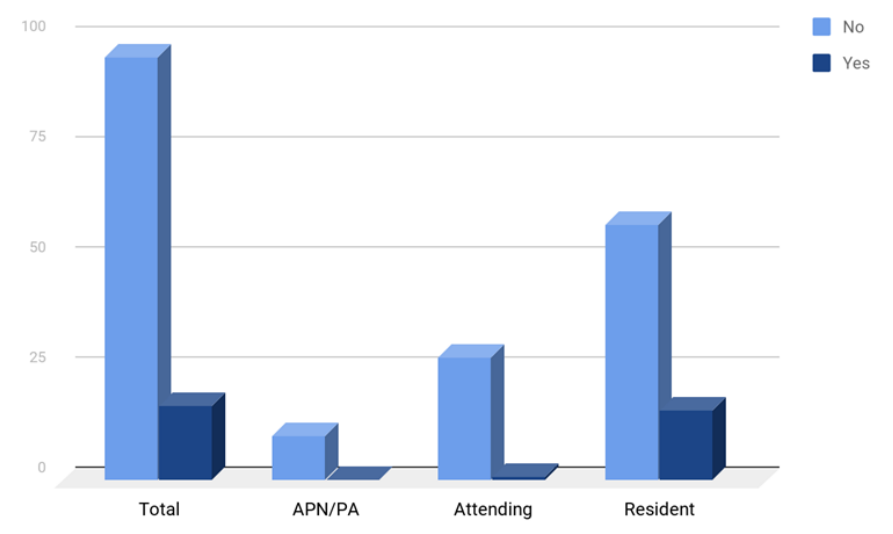

Figure 2 Screening for snoring by provider level of training.

\section{Screening by provider characteristics}

Multivariate analyses were also conducted on several different clinician characteristics. Providers were compared based on level of training, whether attending level, PA, APN, or resident (Figure 2). The resident physician groups were also separated out by number of years in training (Figure 3). Of the 10 patients seen by an APN or PA, none were screened for snoring. Of the 29 patients seen by attending physicians, $1(3 \%)$ was screened for snoring while 28 were not. Among the resident group, 16 of 74 patients (22\%) were screened for snoring $(P=0.029)$. Further categorization of these groups into resident physician vs non-resident physician (attending, PA, APN) revealed that only 1 patient (3\%) was screened for snoring among the non-resident group, as compared with 16 patients $(22 \%)$ in the resident group $(P=0.007)$. The resident physician group was also further investigated based on number of years in training. Of the 74 patients seen by resident physicians, 32(43\%) were seen by first year residents, $24(32 \%)$ by second year residents, $11(15 \%)$ by third year residents, and $7(10 \%)$ by fourth year residents. First year residents screened for snoring in 1 patient (3\%); second year residents in 11 patients $(46 \%)$, third year residents in 4 patients $(36 \%)$, and fourth year residents in no patients $(P=<.001)$. Bivariate analyses were conducted on the progression between each resident year, with the second-year residents more likely to screen for snoring compared to the first-year residents $(P=<.001)$. Screening between second and third year residents and third and fourth year residents was not statistically significant. 


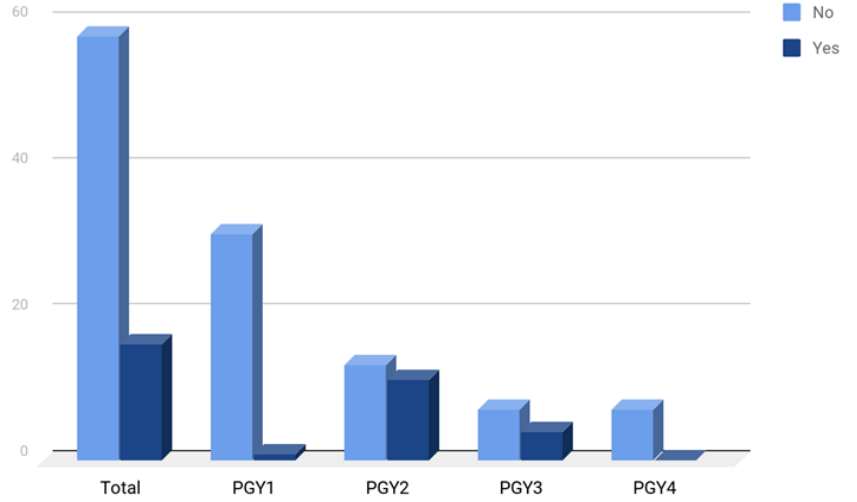

Figure 3 Screening for snoring by resident level of training. PGY indicates Post Graduate Year of training.

\section{Discussion}

This study demonstrates that snoring and OSA are not routinely considered in the differential diagnosis of children presenting with ADHD symptoms, confirming our primary hypothesis. The overall screening rate of $15 \%$ was much lower than we expected given the current literature associating ADHD symptoms with sleep disturbances. It is possible that clinicians were screening for or discussing snoring more frequently during these visits than the data reveal; however, we adopted the standard that if it was not documented, then it did not happen. Among our patient population, we had a realistic distribution ratio of approximately 3:1 between male and female which was consistent with the national incidence of ADHD noted to be around 4:1 for hyperactive subtypes and 2:1 for inattentive subtypes. ${ }^{13}$ While the prevalence of ADHD has been noted to increase with increasing patient age, our study population had a mean age of $7.5 .{ }^{14}$ This is likely due to increasingly early diagnosis, as approximately one-third of patients with ADHD are diagnosed before 6 years of age. ${ }^{15}$ Our population also had the full range of BMI, from 1st percentile to the 99th percentile, with a mean in the upper third quartile. The majority of our patients were diagnosed with the combined subtype, suggesting that they were displaying symptoms of hyperactivity consistent with possible concomitant sleep disturbances. Neither child demographic characteristic was statistically significant in predicting clinician screening rates. Contrary to our hypothesis, those with a BMI greater than the 90th percentile were less likely to be screened for snoring than those without an elevated BMI. While this was not statistically significant, we suspect that we would have observed the same trend if our study had a higher power. Several reasons could contribute to this fact, including clinicians overlooking the BMI measurement in the medical record, inaccessibility of BMI data in the patient's chart, or placing the focus of the visit on dietary and exercise counseling and obesity prevention instead of on screening for OSA.

Furthermore, in a busy pediatric practice, increasing demands are placed on physicians to discuss many aspects of child health, and the association between ADHD symptoms and sleep disturbances may not be at the forefront of the clinician's mind during a particular clinical encounter, even if that child presents with an elevated BMI. Patient gender was also not found to be a significant screening predictor. While multiple studies have demonstrated a higher prevalence of sleep disordered breathing and OSA in males, this did not correlate to an increased rate of screening for snoring in our population. ${ }^{9,16}$ There was widespread practice variation among clinicians in their screening habits for snoring. Both the clinician level of training (resident vs attending vs PA) as well as training year for resident physicians were statistically significant in predicting the clinician's likelihood of screening for snoring. Somewhat surprisingly, among our clinician population, the attending physicians were less likely to screen for snoring than the resident physicians. This was a peculiar finding, given that other research has revealed an increasing suspicion for OSA the longer a particular provider has been in practice. ${ }^{17}$ Attending physicians often have time constraints that residents and APNs/PAs do not have, possibly limiting their ability to either take a thorough history or document said history. Furthermore, it is possible that attending and mid-level providers were in fact screening these children for snoring, but was not documented secondary to time constraints or other unforeseen factors. Also, variation in screening based on resident year was equally fascinating. We did not find an increasing likelihood for questioning based on more time in training, except for the significant increase noted between the first and second year residents. This is likely due to the fact that, at our institution, many second-year residents tend to complete an elective in pulmonary medicine and are educated on this topic. Several factors may contribute to the low screening rate among various providers. Firstly, the average amount of time dedicated to pediatric sleep medicine in medical schools and pediatric residencies is approximately 17 minutes and 4.4 hours, respectively, based on recent studies. ${ }^{18,19}$ Furthermore, OSA is an area where nurse practitioners receive very little training, although curricula can be variable. ${ }^{20}$ Strengths of this study include addition to the current knowledge base regarding screening practices among various healthcare providers with different levels of training, which is less defined than the current literature regarding the epidemiology of snoring, OSA, and ADHD in general. We were interested in examining screening for snoring specifically in the presence of ADHD symptoms, which could lead to an earlier diagnosis of OSA followed by prompt intervention to prevent the chronic sequelae of hypoxia. This study also limitations, most notably its retrospective nature, which predisposes it to potential bias and confounding factors. Moreover, we only screened for snoring and not for other symptoms of sleep disorders such as apnea, enuresis, and daytime fatigue, as examples, which may be present in the absence of snoring in a subset of patients.

Finally, as mentioned previously, it is feasible that snoring could have been included in these visits at a higher rate than indicated by the documentation. Existing evidence suggests that ADHD symptoms can be significantly reduced if the child's underlying OSA is treated with adenotonsillectomy. ${ }^{21}$ Given this fact, interventions designed to increase awareness of sleep disorder related hyperactivity and provider screening practices is a worthwhile pursuit. Potential interventions could include introduction of sleep medicine education earlier in the APN/PA curriculum to increase exposure and hopefully impact future screening practices. Moreover, continued introduction of sleep medicine education to first year residents early on in their training either via didactic lectures or ambulatory based teaching could positively impact screening practices while these young physician habits are still being developed. Furthermore, a screening question about snoring could easily be added to the standardized ADHD questionnaires to encourage parents to include sleep symptoms in the discussion with their doctor and scoring clinicians to include OSA and sleep disordered breathing in their differential diagnosis for patients presenting with hyperactivity. Finally, as the association between hyperactivity, ADHD, snoring, and OSA becomes better recognized 
and established in the literature, routine polysomnography may become part of the routine workup for ADHD. ${ }^{22}$

\section{Conclusion}

The data in this study indicate that snoring and OSA are not routinely considered in the differential diagnosis of children presenting with symptoms of ADHD. Interventions should target evidence-based methods to assist primary care providers in identifying potential OSA related hyperactivity.

\section{Funding details}

No funding was secured for this study.

\section{Acknowledgements}

The authors would like to thank Dr. Yanzhi Wang, statistician at the University Of Illinois College Of Medicine at Peoria for her invaluable assistance with the statistical analysis for this project.

\section{Conflict of interests}

Authors declare that there is no conflict of interest.

\section{References}

1. Wolraich M, Brown L, Brown RT, et al. ADHD: Clinical practice guideline for the diagnosis, evaluation, and treatment of attentiondeficit/hyperactivity disorder in children and adolescents. Pediatrics. 2011;128(5):1007-10022.

2. Froehlich TE, Lanphear BP, Epstein JN, et al. Prevalence, recognition, and treatment of attention-deficit/hyperactivity disorder in a national sample of US children. Arch Pediatr Adolesc Med. 2007;161(9):857864 .

3. Banerjee TD, Middleton F, Faraone SV. Environmental risk factors for attention-deficit hyperactivity disorder. Acta Paediatr. 2007;96(9):1269-1274.

4. Dougherty DD, Bonab AA, Spencer TJ, et al. Dopamine Transporter Density in Patients with Attention Deficit Hyperactivity Disorder. Lancet. 1999;354(9196):2132-2133.

5. Volkow ND, Wang G, Fowler JS, et al. Therapeutic Doses of Oral Methylphenidate Significantly Increase Extracellular Dopamine in the Human Brain. J Neurosci. 2001:21(2):RC121.

6. Bonuck K, Freeman K, Chervin RD, et al. Sleep-disordered breathing in a population-based cohort: behavioral outcomes at 4 and 7 years. Pediatrics. 2012;129(4):e857-e865.

7. Gozal D, Kheirandish-Gozal L. Neurocognitive and behavioral morbidity in children with sleep disorders. Curr Opin Pulm Med. 2007;13(6):505-509.
8. Halbower AC, Mahone EM. Neuropsychological morbidity linked to childhood sleep-disordered breathing. Sleep Med Rev. 2006;10(2):97107.

9. Marcus C, Brooks LJ, Draper K, et al. Diagnosis and Management of Childhood Obstructive Sleep Apnea Syndrome. Pediatrics. 2012;130(3):e714-e755.

10. Honaker SM, Meltzer LJ. Sleep in Pediatric Primary Care: a review of the literature. Sleep Med Rev. 2016;25:31-39.

11. Erichsen D, Godoy C, Granse F, et al. Screening for sleep disorders in pediatric primary care: are we there yet?. Clin Pediatr (Phila). 2012;51(2):1125-1129.

12. Owens JA. The practice of pediatric sleep medicine: the results of a community survey. Pediatrics. 2001;108(3):E51.

13. Rockville MD. Agency for Healthcare Research and Quality. Diagnosis of Attention Deficit/Hyperactivity Disorder Clinical Focus. 1999.

14. Visser SN, Danielson ML, Bitsko RH, et al. Trends in the parent-report of health care provider-diagnosed and medicated attention-deficit/ hyperactivity disorder: United States, 2003-2011. J Am Acad Child Adolesc Psychiatry. 2014;53(1):34-46.e2.

15. Visser SN, Zablotsky B, Holbrook JR, et al. Diagnostic Experiences of Children With Attention-Deficit/Hyperactivity Disorder. Natl Health Stat Report. 2015;3(81):1-7.

16. Goodwin JL, Kaemingk KL, Mulvaney SA, et al. Clinical screening of school children for polysomnography to detect sleep-disordered breathing - the Tucson Children's Assessment of Sleep Apnea study (TuCASA). J Clin Sleep Med. 2005;1(3):247-254.

17. Honacker SM, Dugan T, Daftary A, et al. Unexplained Practice Variation in Primary Care Providers' Concern for Pediatric Obstructive Sleep Apnea. Acad Pediatr. 2018;18(4):418-424.

18. Mindell JA, Bartle A, Ahn Y, et al. Sleep education in pediatric residency programs: a cross-cultural look. BMC Res Notes. 2013;6:130.

19. Mindell JA, Bartle A, Wahab NA, et al. Sleep education in medical school curriculum: a glimpse across countries. Sleep Med. 2011;12(9):928-931.

20. Mindell JA, Owens JA. Sleep problems in pediatric practice: clinical issues for the pediatric nurse practitioner. $J$ Pediatr Heal Care. 2003;17(6):324-331.

21. Chervin RD, Ruzicka DL, Giordani BJ, et al. Sleep disordered breathing, behavior, and cognition in children before and after adenotonsillectomy. Pediatrics. 2006;117(4):e769-e778.

22. Silvestri R, Gagliano A, Arico I, et al. Sleep disorders in children with Attention-Deficit/Hyperactivity Disorder (ADHD) recorded overnight by video-polysomnography. Sleep Med. 2009;10(10):1132-1138. 